\title{
Modeling and simulation in the design process of a prosthetic foot
}

\author{
Heimir Tryggvason ${ }^{1} \quad$ Felix Starker $^{2} \quad$ Christophe Lecompte $^{2} \quad$ Fjóla Jónsdóttir $^{1}$ \\ ${ }^{1}$ School of Engineering and Natural Sciences, University of Iceland, Iceland, \{het $\left.11, \mathrm{f} j\right\}$ ahi. is \\ Össur hf, Iceland, fstarker@ossur.com, clecomte@ossur.com
}

\begin{abstract}
The design process of prosthetic feet largely depends on an iterative process of prototyping and user testing. As resources for reliable and repeatable user testing are limited, modeling and simulated testing of the design is a positive addition to this process to support further design development between prototyping.

The key goal of prosthetic foot design is to mimic the function of the lost limb. A passive spring and damper system can imitate the behavior of an ankle for low level activity, e.g. walking at slow to normal speeds and relatively gentle ascents/descents. In light of this, a variety of constant stiffness prosthetic feet are available on the market that serve their users well. However, when walking at a faster pace and ascending/descending stairs, the function of the physiological ankle is more complex and the muscular activity contributes to the stride in different ways.

One of the challenges in prosthetic device design is to achieve the appropriate range of stiffness of the arrangement of joints and spring elements for different tasks, as well as varying loading of the prosthetic device. This calls for an adaptive mechanism that mimics the stiffness characteristics of a physiological foot by applying real-time adaptive control that changes the stiffness reactively according to user's needs. The goal of this paper is to define the stiffness characteristics of such a device through modeling.

A finite element model was made for a well-received prosthetic foot design. The model was then validated by mechanical measurements of the actual product. We further enhanced the model to include a secondary spring/dampener element to provide added flexibility and damping of the ankle joint movement. Reactive control of the secondary element allows the simulated prosthetic foot to adapt the ankle joint to imitate the behavior of the physiological ankle during different activities and in different phases of the gait cycle.
\end{abstract}

\section{Introduction}

The goal of all prosthetic design is to mimic as closely as possible the actual physiological look, function and feel of the replaced body part. A real foot and ankle joint is a complex system of muscles, sinews and ligaments, whose functionality is challenging to replicate. Many current prosthetic feet are mechanical designs with fixed spring and dampening characteristics, optimized for rather slow gait on a level surface (Hansen, 2004). However, such devices are not optimal for many everyday tasks, such as ascending and descending stairs, walking on uneven terrain and running. Higher ankle joint stiffness is required when walking rapidly or running, while a softer spring provides more comfort when walking slowly and places less strain on the residual limb. Changing prosthetics or making frequent manual adjustments is cumbersome. Adaptive prosthetics that suit a range of activities provide a better quality of life for the user. The human ankle and limb adapts dynamically to the terrain and variations in pace (Jin, 2016; Hunter, 2010). This calls for feet that have real-time variable stiffness.

Research on human legs and gait have produced many different designs of prosthetic feet. They can be divided into three main categories: conventional feet, energy storing feet and motor powered feet. A conventional foot is a solid ankle cushioning heel, which does not follow a normal gait pattern. Energy storing feet, such as, Vari-Flex ${ }^{\circledR}$ and Pro-Flex ${ }^{\circledR}$ are made of a layered carbon-fiber composite that extends from the amputees' residual limb and aims to provide natural progression through the stages of normal gait. The stiffness of the foot, and its response to load, may be controlled via the thickness profile of the foot, as well as its shape. Additionally, a spring may be introduced to the structure to provide further shock absorption. In the third category are powered active prosthetic ankles, such as bionic ankle-foot prosthesis (Herr, 2012; Au, S., 2008) and the Proprio ${ }^{\circledR}$ foot. The Proprio foot is an adaptive prosthetic device for low to moderately active below-knee amputees that mimics natural foot motion. Quasi-passive feet (Williams, 2009; Jin, 2016; Li, 2006) are a further evolution in prosthetics design, whose goal is to adapt more closely to the users' needs.

Determining the optimal characteristics of a prosthetic foot in the design phase is still a process of trial and error and relies highly on the prosthetic users' feedback during real life tests. Our approach is to use 
mechanical testing and finite element (FE) analysis to optimize the stiffness characteristics of a prosthetic foot. The Pro-Flex ${ }^{\circledR}$ prosthetic foot by Össur, Iceland was selected for the analysis as it is a popular example of a passive prosthetic design. The mechanical characteristics were determined using a dynamic test machine, according to ISO 16955. The standardized testing can be viewed as a step between simulations and real life user tests that provides consistent results and a less complex way of understanding and improving the characteristics of prosthetic feet. A model was constructed in the commercial finite element software ANSYS, whose purpose is to serve as a framework for experimenting with different material behavior, as well as additional elements, to improve the characteristics of the prosthetic design. Both static and dynamic simulations were carried out. The simulation results were validated by comparison with the measured mechanical characteristics. The modeled results were found to accurately match the experimental ones, in terms of deformation of the carbon fiber spring blades, as well as the resulting anterior and posterior forces and ankle moments. The finite element (FE) model was used to explore several variations of the foot design, including varying spring stiffness and dampener elements. The end goal is to use this model to experiment with actively controlled elements that adapt dynamically to the users' needs.

\section{The Subject Prosthetic Foot}

The Pro-Flex ${ }^{\circledR}$, Cat. 5, size 27 was selected as a prosthetic foot model for this study; see Figure 1. One of the features of the design is a mechanical pivot within the composite springs, allowing for a defined ankle rotation. A mechanical link element is located posterior, connecting the midfoot spring with the pyramid adapter that is attached to the user's leg.

To change the stiffness characteristics of this foot the mechanical link seems as an ideal part as it influences both the rotation around the pivot and interacts with the mid blade spring.

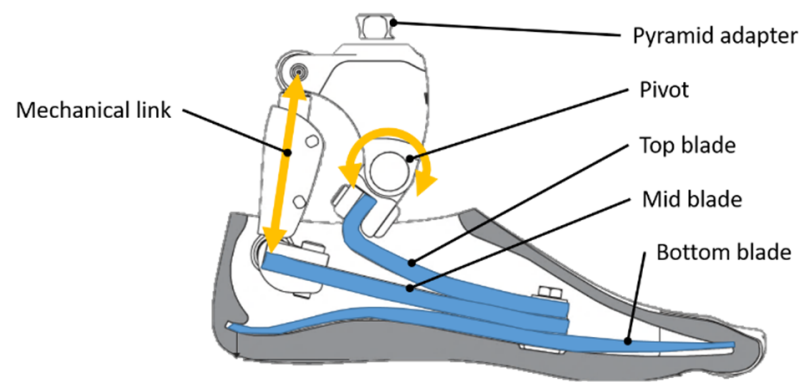

Figure 1: Schematic of the prosthetic foot model used for all tests (Pro-Flex ${ }^{\circledR}$ Össur).

\section{Methodology}

Subject based data is highly variable and the prosthetic user continuously adapts its motion profile. The approach for this study is to use a combination of mechanical testing and finite element analysis as described schematically in Figure 2. Mechanical testing of an actual prosthetic is first carried out. A simulation model is constructed and the prosthetics characteristics as obtained by testing, used to parametrize and validate the model. After completion of this first validation, the foot model is modified by introducing non-linear materials, e.g. spring and damper, to a specific section of the prosthesis to allow for an altered foot performance. The optimized models will later on be transferred into physical prototypes and finally fit to prosthetic users.

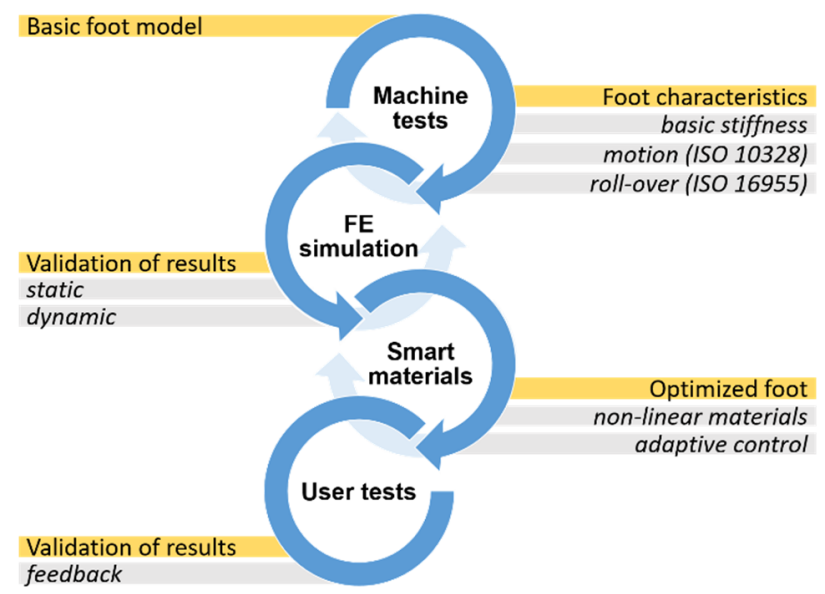

Figure 2: The methodologic approach for validating the simulation model of a prosthetic foot.

The basic foot model is setup by investigating the isolated stiffness characteristics of the carbon fiber spring blades for heel and toe by an internal standardized test procedure (basic stiffness). ISO 10328 a simplified dynamic endurance test is used as a first validation. In addition, ISO 16955 is used to simulate more realistic a complete heel to toe roll-over of the foot.

Current works investigate the links between actual user experience and these standardized tests. Hence, if the simulation model closely matches the mechanical testing, we can conclude that the model is capable of accurately describing the actual prosthetic users' experience.

\section{Mechanical Testing}

Stiffness evaluation of the foot sample was performed with a uniaxial tension and compression test machine (M350-10CT, Testomatic, UK) with linear displacement and constant speed of $300 \mathrm{~mm} / \mathrm{min}$. The foot sample is rigidly connected to the machine and set 
under $15^{\circ}$ angle for the heel and $15^{\circ}$ respectively for the toe test. With $36 \mathrm{~mm}$ of set displacement, a resulting vertical force of $610+/-35 \mathrm{~N}$ was measured for the toe. Respectively, a $12 \mathrm{~mm}$ set displacement resulted in 923 $+/-45 \mathrm{~N}$ for the heel spring, measured throughout 40 samples. The variation between samples is due to variations in the carbon fiber blade production.

The mechanical testing of the prosthetic foot took place with two approaches. ISO 10328 is used for endurance testing and has a simplified setup with a prosthetic foot connected rigidly to the machine frame. Two pistons are compressing heel and toe with a defined phase shift and applying the dynamic load at a defined angle $\left(-15^{\circ}\right.$ heel and $+20^{\circ}$ toe), see Figure 3 .

Although ISO 10328 is valid for strength testing, deformation and motion data cannot be linked to prosthetic user experience due to the limited rigid setup.

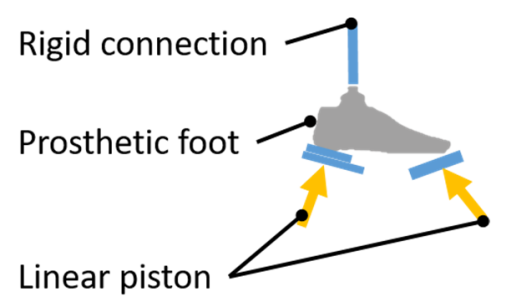

Figure 3: Schematic of the static loading according to ISO 10328.

Therefore, a second test was carried out with a modified test machine (Shore Western, USA) to evaluate the characteristics of the prosthetic foot under dynamic loading conditions, including swing phase according to ISO 16955; see Figure 4.

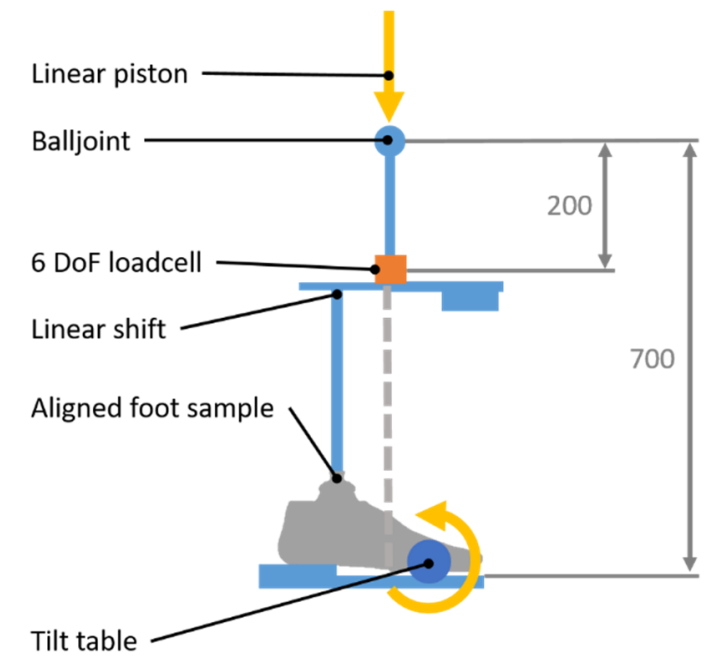

Figure 4: Schematic of the dynamic loading according to ISO 16955.

For this study, the focus is on the heel-to-toe roll-over test, applying a dynamic force of $824 \mathrm{~N}$ maximum, synchronized to a rotational motion ranging from $-20^{\circ}$ to $+40^{\circ}$ angle during $0.6 \mathrm{~s}$ of stance phase; see Figure
5. The foot suspension is achieved by a ball joint with low spring force $(14 \mathrm{~N})$ to reset the foot during swing phase. Hence, the foot is capable of rolling and deforming freely under the set load profile.

Several sensors capture the forces, the resulting torque and the displacement within the prosthetic leg. The horizontal rotational tilt table is in constant displacement control, as well as the linear piston pushing on the prosthetic foot. The force is adjusted by a PI-loop controller with the force profile as feedback signal; see Figure 5.

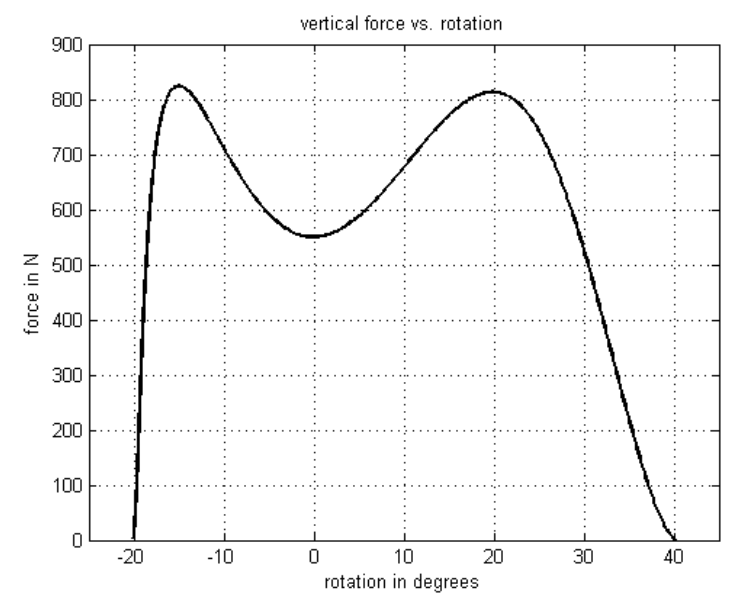

Figure 5: Input data (vertical force vs. the rotation of the tilt table) for the test machine.

\section{Finite Element Model}

A 3D FE model was built and simulated in Ansys WB; see Figure 6. In the FE model, the carbon blades are modeled as flexible surface bodies and assigned a layer element designating the buildup of the carbon layers; thickness, layer angle and material of the different layers in the section. Each part is also assigned an orientation element to designate the orientation of the carbon layers. The material properties used for modeling the carbon fiber blades are a combination of values provided by the supplier of the pre-preg carbon fibers and measurements made on carbon fiber plates prepared in a production line.

All other parts of the foot are simplified to four rigid bodies in the model, that is, main body (Pyramid adapter), mechanical link and two blade clamps. These are made of aluminum alloy and steel and are considered stiff enough to neglect deformation. The three joints between the rigidly modeled bodies have a rotational degree of freedom about an axis, at each connection of the mechanical link and at the pivot connection of the top blade to the main body. The connection between the three flexible surface bodies and connection of the clamps to the top and middle blade are modeled with contact elements in the area of the bolted clamping. These contact areas are the most sensitive attribute of the model where contact stiffness affects the 
deformation of the flexible blades and therefore the overall quasi stiffness of the modelled foot.

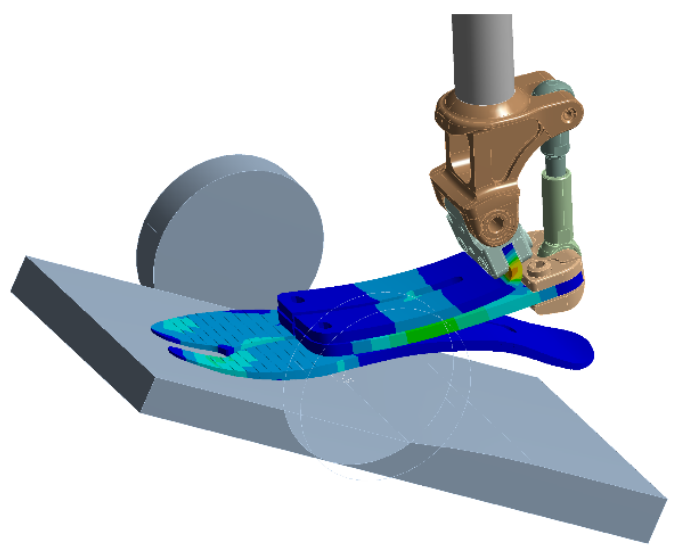

Figure 6: 3D view of the FE model of the Pro-Flex in ISO16955 test simulation, showing equivalent stresses in flexible parts.

The force reaction in the mechanical link goes from compression to tension, transitioning from heel-strike to toe-off. The maximum tension in the mechanical link is roughly three times the ground reaction force at maximum force on the fore-foot. Hence, effects of changes to the function of the link will influence the gait more after mid-stance than at heel-strike.

This originally rigid link allows for a superimposed spring and damping characteristics altering the rotational stiffness of the foot during roll-over. The modelling of the test procedure is aimed at investigating the effects of changed function in this connection to approach a more desirable overall effect in the response of the foot.

Initial validation of the FE model was done by simulating the ISO 10328 test on the foot assembly. Force values were acquired from remote displacement boundary condition on a rigid plate at the appropriate angle on both toe and heel of the foot model. The resulting quasi stiffness on toe and heel then compared to measured values from the mechanical testing. Free frictional sliding contact was defined between elements on the sole blade and the rigidly defined plate to ensure correct rollover on the sole blade for the ramped displacement.

Furthermore, simulation was done to validate the model against the ISO 16955 test procedure. The tilt table and linear shift geometry was modeled as rigid bodies and a transient structural analysis performed with test machine input data as boundary conditions for the simulation; see Figure 7. For this simulation, the contact between tilt table and sole blade is defined as fully rough, with no slippage allowed. Values from the simulation are compared to test machine output data, that is, the vertical displacement of the ball joint, the force reaction and the moment at knee level.

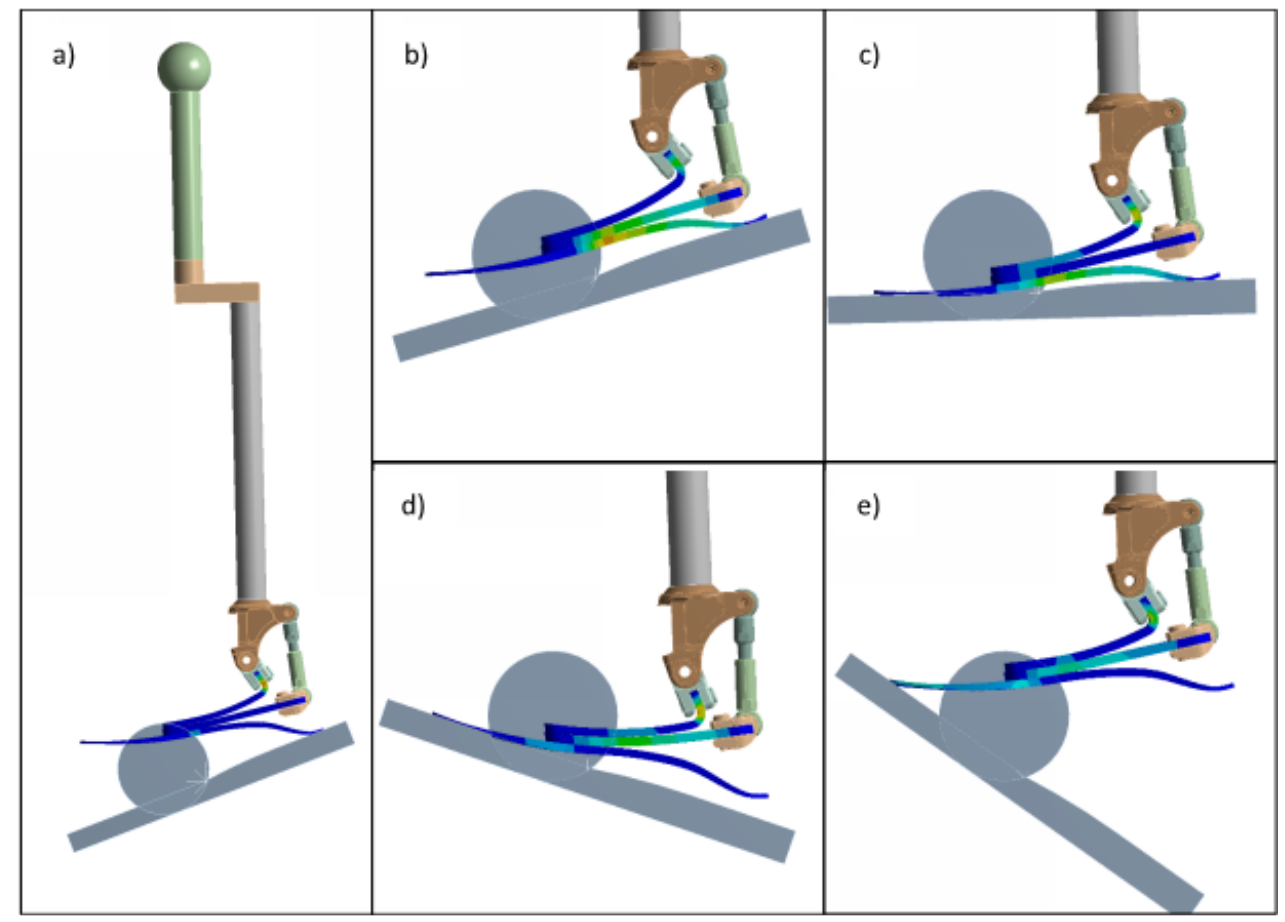

Figure 7: Transient simulation of the ISO 16955 test procedure for a $600 \mathrm{~ms}$ loop. a) Showing full model at early heelstrike, at $10 \mathrm{~ms}$. b) Mid heel strike at max. vertical force, $150 \mathrm{~ms}$. c) Mid-stance, at $300 \mathrm{~ms}$. d) Right after heel-off, at 450 ms. e) Before Toe-off at $575 \mathrm{~ms}$. 


\section{Model Validation}

The machine test according to ISO 16955 and simulation showed comparable results during a full rollover of the prosthetic sample, as shown in Figure 8. For the initial set parameters of the load vs. rotation profile, an accuracy of better than $+/-1 \%$ of the maximum force value of $824 \mathrm{~N}$ was achieved at the given stance phase time of $600 \mathrm{~ms}$.

The resulting moment, measured at $500 \mathrm{~mm}$ above the tilt table, which represents the position of the knee joint, see figure 4 , showed similar characteristic curves. The transition of the foot from the deformed heel to toe occurred at the same angle, which shows similar motion of the overall foot. A slight deviation of the resulting moment, especially after $-15^{\circ}$ to $-2^{\circ}$, was found.

The quasi stiffness calculated by the vertical force divided by the vertical displacement showed high correlation until late stance $\left(26^{\circ}\right.$ to $\left.40^{\circ}\right)$ where the foot only rests on the very toe-end of the bottom blade. Peak values for both knee moment and stiffness ranged within $+/-2 \%$ of the peak value.

The overall correlation between measurements and simulation are within required parameters for a valid comparison and valuation of shifts in the curves as the function of the mechanical link is changed.

\section{Design Enhancements}

The basic foot model as described above, was extended, allowing experimentation with the effects of modifying design elements. This work focuses on substituting a spring and dampener element for the rigid mechanical link shown in figure 1. Our hypothesis is that a translational link allows for a greater range of motion that can give increased comfort for the amputee. The results of the modeling of the enhanced design are shown in Figure 9. Initial parameters for spring stiffness and dampening for the translational joint are chosen as a moderate change for controlled adjustment steps towards a modified function i.e. a relatively high spring constant and low damping coefficient. $(\mathrm{k}=200 \mathrm{kN} / \mathrm{m}, \mathrm{c}$ $=10 \mathrm{kN} \mathrm{s} / \mathrm{m}$ ).

The input vertical force vs. plate angle profile for both the simulation and the test machine remains constant. Therefore, it can be assumed that all following data such as knee moment and quasi-stiffness is comparable to the initial comparison test. The sagittal moment at the knee transitions later from heel to toe. Resulting peak moment at late stance can be reduced by $15 \%$. The quasi stiffness curve shows similar characteristics with a reduced peak stiffness at heel strike of $8 \%$ and a softer late stance. This can be explained by the higher range of motion in the pivot within the prosthetic foot model and a deflection of the spring and damper system the foot motion is altered. The foot performs in late stance softer compared to the foot with a rigid link.

Comparing all data shows that the modified prosthetic foot does not evenly change its stiffness as it would by using softer carbon fiber spring blades. The spring-damper system in combination with the carbon fiber springs alters the overall transition and mechanical behavior of the foot which is well received during stair ambulation, when walking over slopes or at slow walking speeds.
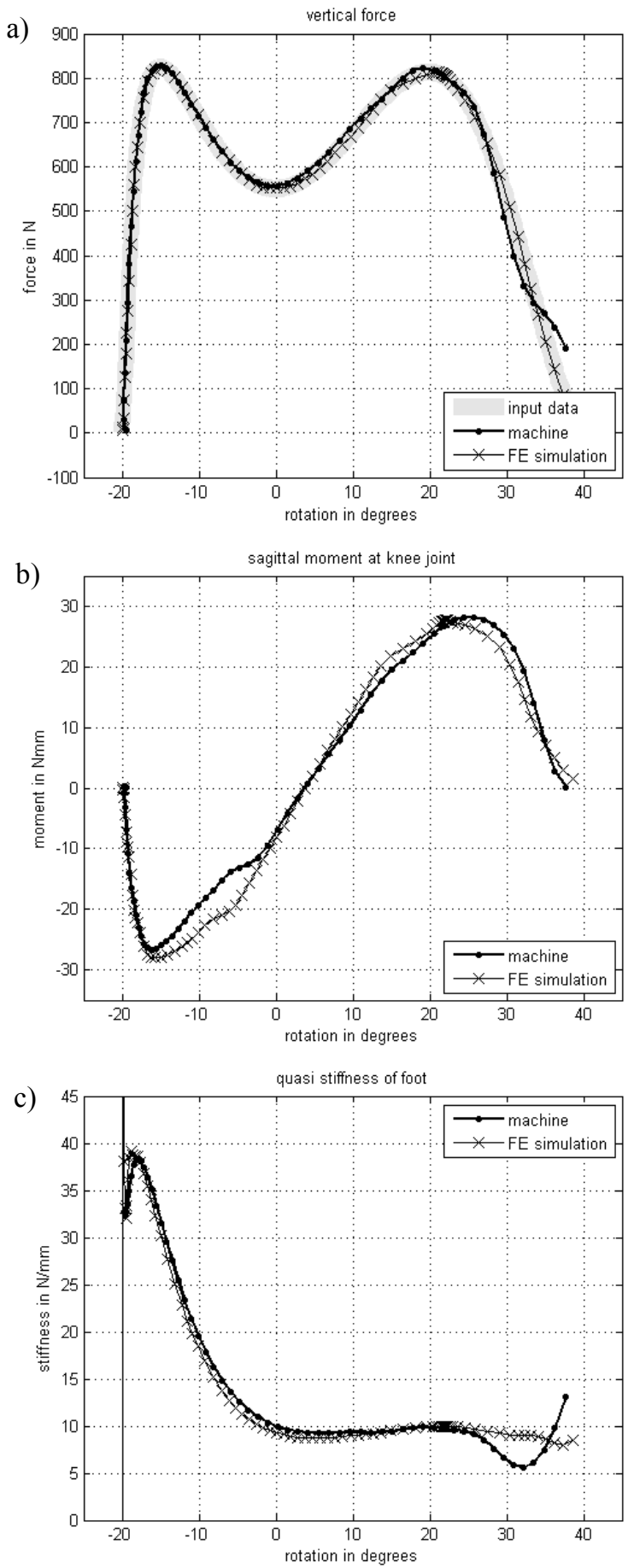

Figure 8: Results for the original Pro-Flex ${ }^{\circledR}$.

Measurements of the 16955-test machine in comparison 
to the simulation a) vertical force vs. rotation angle or the plate b) knee moment c) quasi stiffness.

This alteration is less favorable for higher activity levels, e.g. walking at higher pace and running. The function needed in the link is to allow the flexibility at slower speeds but reduce it at higher speed.
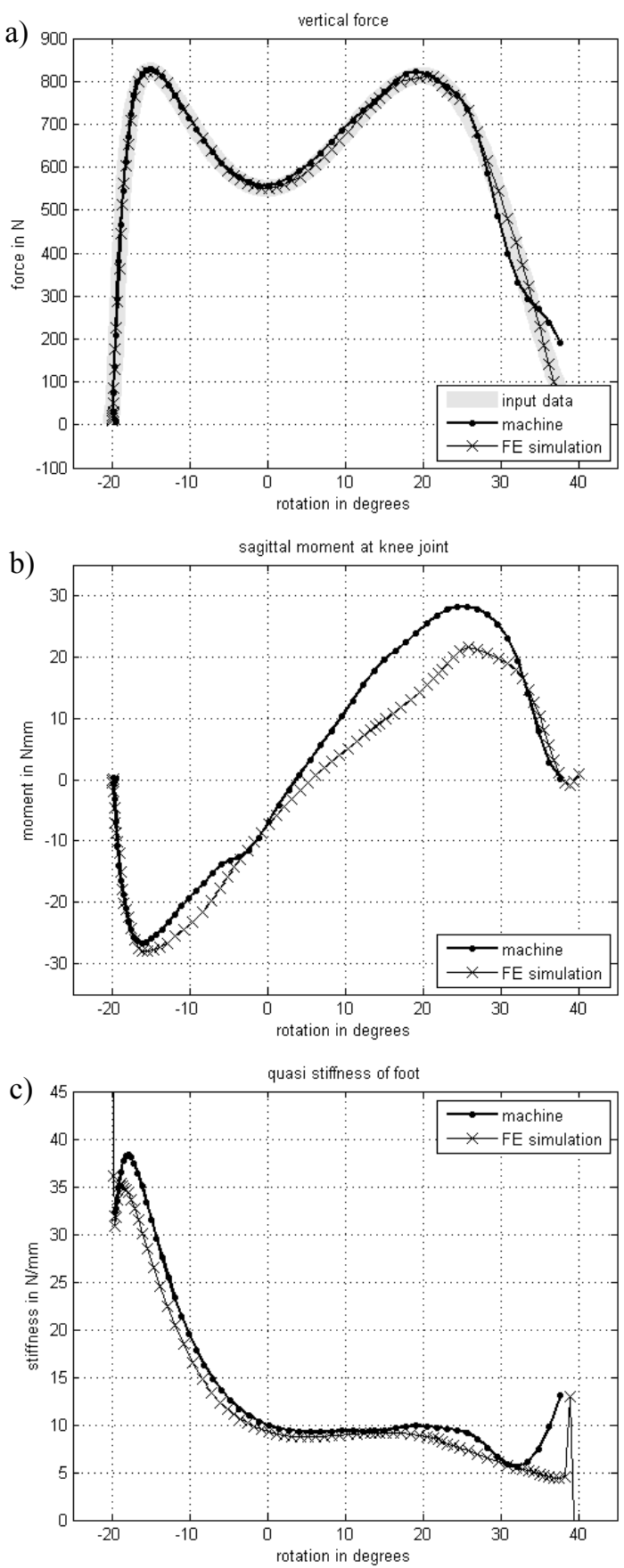

Figure 9: Comparison between measurements of the 16955-test machine and the simulation of a modified model a) vertical force vs. rotation angle or the plate b) knee moment c) quasi stiffness

Beside the overall good correlation of the results with the presented approach, minor offset and differences can be explained by the absence of the effects of a cosmetic foot cover within the simulation. The foot cover provides a softer heel strike with a smooth transition towards midstance. At late stance, the foot cover elongates the foot by transitioning the load from the carbon fiber bottom blade to the elastic toes. All these effects can directly be examined in the comparison figures.

Apart from this the foot is under minimal unwanted sliding conditions, during the machine test, especially when the load is removed of the sample during late stance. Throughout this, each sensor, actuator and data acquisition of the machine causes a potential error that is by calibration assured to be below $1 \%$ of the full signal, nevertheless a range of uncertainty remains. Altogether, this clearly points out that the simulated approach shows equivalent results and by this is highly suitable for further improvements of the prosthetic foot design and too evaluate the stiffness characteristics.

\section{Conclusions}

The study has shown that the standardized test procedure can be simulated and modifications to the model show anticipated shifts in function. Further work is aimed at modeling the desired shift in function of the mechanical link for different displacement rates and design of a functional link.

Ongoing research has to prove the links between these mechanical characteristics and different groups or the individual amputees. Nevertheless, the FE simulation model showed high correlation with the machine test results. Some differences can be explained by the use of a cosmetic cover within the machine test to allow for a defined friction. The FE model seems highly capable to optimize the application of new nonlinear function in the foot. Comparison and interpretation of simulation results against standardized test machines is more practical than to traditional gait data. Further studies have to connect prototypes to the user experience and close the methodological development loop.

\section{Acknowledgements}

This work is funded by the Technology Development Fund, Rannis, Iceland.

\section{References}

Ahmadkhanlou F., Zite, J. and Washington, G., 2007, "A magnetorheological fluid-based controllable active knee brace", Smart Structures and Materials Proc. SPIE 6171 pp. 148-56.

$\mathrm{Au}, \mathrm{S} .$, Berniker, M. and Herr. H., 2008, "Powered ankle-foot prosthesis to assist level-ground and stair-descent gaits", Neural Networks, Vol. 21, pp. 654-666.

C. Li, M. Tokuda, J. Furusho, K. Koyanagi, S. Morimoto, Y. Hashi-moto, A. Nakagawa, and Y. Akazawa, 2006, " Research and development of the intelligently controlled 
prosthetic ankle joint," in Proc. IEEE Int. Conf. Mechatronics and Automation, Luoyang,China, pp.11141119.

Deffenbaugh, B. W., Herr, H. M., Pratt, G. A. and Wittig, M. B., 2004, "Electronically controlled prosthetic knee" US Patent Specification 6,764,520.

Gudmundsson, K. H., Jonsdottir, F. and Thorsteinsson, F., 2010, "Geometrical optimization of a magneto-rheological rotary brake in a prosthetic knee", Smart Materials and Structures, Vol. 19, 035023

Hansen, A. H., Childress, D. S., Miff, S. C, Gard, S. A. and Mesplay, K. P., 2004, "The human ankle during walking: Implications for design of biomimetic ankle prosthesis", Journal of Biomechanics, Vol. 37, pp. 1467-1474.

Herr, H. M. and Grabowski, A. M., 2012, "Bionic ankle-foot prosthesis normalizes walking gait for persons with leg amputation”, Proc. R. Soc. B., Vol. 279, pp. 457-464.

Herr, H., and Wilkenfeld, A., 2003, "User-adaptive control of a magnetorheological prosthetic knee. Industrial Robot: An International Journal, Vol. 30, pp. 42-55.

Hunter, L. C., Hendrix, E. C., \& Dean, J. C., 2010, “The cost of walking downhill: Is the preferred gait energetically optimal?", Journal of Biomechanics, 43(10), pp.1910-1915.

ISO/TC 168 Prosthetics and orthotics. (2016). ISO 10328:2006 Prosthetics -- Structural testing of lower-limb prostheses -- Requirements and test methods. ISO. Retrieved from: https://www.iso.org/standard/36413.html

ISO/TS 16955:2016 Prosthetics - Quantification of physical parameters of ankle foot devices and foot units. (2016). Retrieved from: https://www.iso.org/standard/69821.html

Jin, L., Adamczyk, P. G., Roland, M., \& Hahn, M. E. (2016). "The effect of high- and low-damping prosthetic foot structures on knee loading in the uninvolved limb across different walking speeds", Journal of Applied Biomechanics, Vol. 32(3), pp. 233-240.

Williams, R. J., Hansen, A. H. and Gard, S. A., 2009, "Prosthetic ankle-foot mechanism capable of automatic adaptation to the walking surface", J. Biomech. Eng., Vol. 131, pp. 035002-1-7.

URL: http://www.ansys.com

URL: http://www.ossur.com/prosthetic-solutions 